\title{
EphrinA I-targeted nanoshells for photothermal ablation of prostate cancer cells
}

\author{
Andre M Gobin \\ James J Moon \\ Jennifer L West
}

Department of Bioengineering, Rice University, Houston, TX, USA
Correspondence: Jennifer L West Department of Bioengineering, Rice University, PO Box 1892, MS I42, Houston, TX 7725I-1892, USA

$\mathrm{Tel}+\mathrm{I} 7 / 33485955$

$\mathrm{Fax}+|7| 33485877$

Email jwest@rice.edu

\begin{abstract}
Gold-coated silica nanoshells are a class of nanoparticles that can be designed to possess strong absorption of light in the near infrared (NIR) wavelength region. When injected intravenously, these nanoshells have been shown to accumulate in tumors and subsequently mediate photothermal treatment, leading to tumor regression. In this work, we sought to improve their specificity by targeting them to prostate tumor cells. We report selective targeting of PC-3 cells with nanoshells conjugated to ephrinA1, a ligand for EphA2 receptor that is overexpressed on PC-3 cells. We demonstrate selective photo-thermal destruction of these cells upon application of the NIR laser.
\end{abstract}

Keywords: nanoshell, near infrared, photothermal treatment, prostate cancer

\section{Introduction}

Nanoshells are a class of nanoparticles consisting of an ultrathin metal shell surrounding a dielectric core (Averitt et al 1999). Gold-coated silica nanoshells can be designed to possess strong absorption of light in the near infrared (NIR) wavelength region between $650-950 \mathrm{~nm}$; this is significant for biomedical applications as tissue components have minimal absorption at these wavelengths (Weissleder 2001). Gold nanoparticles are well suited for use in medicine due to the biocompatibility of gold; studies have shown no cytotoxicity of gold particles (Connor et al 2005). In addition, the gold surface allows easy conjugation of biomolecules to the surface by the use of a poly(ethylene glycol) (PEG) linker with a sulfur moiety (Loo et al 2005a; Lowery et al 2006). NIR-absorbing nanoshells have been used for cancer therapy (Hirsch et al 2003; Lowery et al 2006), showing up to $100 \%$ regression of tumors after photothermal treatment (O’Neal et al 2004). These studies employed nontargeted nanoparticles that accumulated in the tumor tissues due to the enhanced permeability and retention (EPR) effect. It may be possible to achieve high efficacy at lower nanoparticle dosages and also minimize nonspecific effects using targeted nanoparticles.

Eph receptors and ephrin ligands, a tyrosine kinase family of proteins, have been implicated in many types of cancers, including prostate, lung, esophageal, melanoma, leukemia colorectal, cervical, ovarian, and breast cancers (Surawska et al 2004; Brantley-Sieders et al 2004). Eph/Ephrin signaling plays an essential role in formation and maintenance of tissue organization by regulating cell adhesion, repulsion, and migration (Miao et al 2000; Marston et al 2003; Poliakov et al 2004). Abnormal Eph/Ephrin signaling activities and thus loss of cell-cell organization in tumors may be an important reason that tumors with high levels of expression of Eph receptor are more apt to metastasize and have poor prognosis (Fang et al 2005). In particular, a subclass of transmembrane Eph receptors, EphA2, has been shown to be overexpressed in prostate, melanoma, breast, and lung cancers (Walker-Daniels et al 1999; Zelinski et al 2001; Hess et al 2001; Brantley et al 2002; Kinch et al 2003; Ireton and Chen 2005). 
Metastatic prostate tumor cell lines, including LNCaPs and PC-3 cells, overexpress EphA2 by $10-100$-fold as compared to noninvasive prostatic epithelial cells (Walker-Daniels et al 1999). Expression levels of EphA2 are known to be progressively increased in more aggressive prostate cancer cell lines (Fox and Kandpal 2004; Fox et al 2006). In addition, EphA2 immunoreactivity is significantly greater in malignant human prostate carcinoma compared to benign prostate epithelium (Walker-Daniels et al 1999), and progressively higher levels of EphA2 were found in highgrade prostatic cancers (Zeng et al 2003). Thus, it has been suggested that expression levels of EphA2 are correlated with disease stage, tumor metastasis, and poor prognosis (Ireton and Chen 2005). EphA2 receptor can be targeted by its natural ligand ephrinA1 or anti-EphA2 antibody. Once targeted by these moieties, EphA2 receptor becomes activated and starts signaling events that lead to inhibition and reversal of malignant phenotypes, such as proliferation, migration, and invasiveness (Carles-Kinch et al 2002; Zelinski et al 2001).

In this current study, we aimed to target and destroy prostate cancer cells by targeting them with nanoshells conjugated to ephrinA1, a natural ligand for EphA2 receptor. We have confirmed increased expression level of EphA2 receptor in a model prostate cancer cell line, PC-3. When NIR-absorbing nanoshells were conjugated with ephrinA1, they specifically bound to PC-3 cells, and subsequent photothermal therapy with NIR light selectively killed PC-3 cells, demonstrating the efficacy of targeted nanoshell therapy.

\section{Methods and materials}

All chemicals were purchased from Sigma-Aldrich (St. Louis, MO, USA) unless otherwise noted.

\section{Nanoshell synthesis}

Gold nanoshell synthesis has been previously described by others (Oldenburg et al 1998). Briefly, nanoshells were made through a four-step procedure. First, silica cores were grown using the Stöber process, the basic reduction of tetraethyl orthosilicate (TEOS). $45 \mathrm{ml}$ of 200-proof ethanol was used with $3.0-4.0 \mathrm{ml}$ of $14.8 \mathrm{~N} \mathrm{NH}_{4} \mathrm{OH}$ to make six batches at different ammonia volumes. $1.5 \mathrm{ml}$ TEOS was added to each batch and allowed to react a minimum of $8 \mathrm{hr}$. Silica precipitates were centrifuged and washed with 200-proof ethanol twice to remove any remaining $\mathrm{NH}_{4} \mathrm{OH}$. The resultant silica nanoparticles were sized using scanning electron microscopy (SEM; Philips FEI XL30). Average diameters of different batches ranged between 98-112 nm. Only batches with a polydispersity of less than $10 \%$ were used in subsequent steps. Reaction of the silica core nanoparticles with $200 \mu$ of (3-aminopropyl) triethoxysilane (APTES) per batch provided amine groups on the surface of the cores to allow for adsorption of gold colloid in the subsequent step. Aminated silica cores were boiled for $2 \mathrm{hr}$ with addition of 200-proof ethanol to maintain volume, then cooled and washed twice by centrifugation. The silica core suspensions were measured to determine the weight percent of solids and adjusted to $4 \mathrm{wt} \%$ for storage by addition of ethanol. Next, gold colloid was prepared to a size of $2-4 \mathrm{~nm}$ as by the method of Duff and colleagues (1993) and aged 2-3 weeks at $4{ }^{\circ} \mathrm{C}$. The colloid was then concentrated $\sim 20 \times$ through rotary evaporation and mixed with the aminated silica particles at a volume of $10 \mathrm{ml}$ concentrated colloid:300 $\mu 1$ stored silica core suspension; thus allowing small gold colloid to attach to the larger silica nanoparticle surface to act as nucleation sites in the subsequent reduction step. This resulted in the seed particles form which nanoshells are grown. Seed particle suspensions were adjusted to have an absorbance at $530 \mathrm{~nm}=0.1$.

Finally, the gold shell was then grown by reduction of gold using $0.4 \mathrm{mM} \mathrm{HAuCl}_{4}$ solution (plating solution) in the presence of formaldehyde. To produce particles with varying shell thicknesses, we varied the concentration of seed particles while using the same amount of plating solution. NIR absorption characteristics of the nanoshells were determined using a UV-Vis spectrophotometer (Carey 5000 Varian, Walnut Creek, CA). Samples with the appropriate NIR peak resonance $(\sim 800 \mathrm{~nm})$ were scaled up linearly to provide nanoshells for the experiment.

\section{EphrinAl conjugation to nanoshells}

A bi-functional PEG derivative, orthopyridyl-disulfidepoly(ethylene glycol)-N-hydroxysuccinimide ester (OPSS-PEG-NHS, 2000MW) was obtained from Nektar (Birmingham, AL, USA). The NHS group is cleaved in water, leaving an activated carboxylic group, which reacts with a primary amine in biomolecules to form amide bond between the PEG chain and biomolecules of interest. A disulfide group in the OPSS end allows self-assembly of the biomolecules conjugated to the PEG chains on the gold surface of nanoshells. Recombinant fusion proteins of mouse ephrinA1/Fc chimera (R and D Systems, Minneapolis, $\mathrm{MN}, \mathrm{USA}$ ) were reacted with bi-functional PEG at a mole ratio of 20:1. Reactants were dissolved in $100 \mathrm{mM}$ sodium bicarbonate at $\mathrm{pH}$ 8.5. The final concentration of the OPSS-PEG-ephrinA1 conjugate was approximately $8.25 \mu \mathrm{M}$, 
and the product was used to bind to the gold surface of the nanoshell. OPSS-PEG-ephrinA1 was incubated at a ratio of 1500 molecules per nanoshell for $4 \mathrm{hr}$ to allow self-assembly of the disulfide end of the molecule to the gold surface. Nanoshells were then further coated with PEG to block any remaining uncovered gold surfaces. This was accomplished by adding $20 \mu \mathrm{l}$ of $5 \mu \mathrm{M}$ of PEG-thiol (Nektar, Huntsville, AL, USA) to $1.5 \times 10^{10}$ nanoshells/ml in DI water. Control PEG-nanoshells were synthesized in parallel by binding PEG-thiol to the gold surface of the nanoshells. Conjugated nanoshells were sterilized by filtration using a $0.22 \mu \mathrm{m}$ filter. Extinction spectra of ephrinA1-, PEG- and bare nanoshells were obtained from $400 \mathrm{~nm}$ to $1100 \mathrm{~nm}$ with Carey UV-Vis spectrophotometer.

\section{Cell culture}

Human prostate cancer cells, PC-3 cells, obtained from ATCC, were grown in Ham's F12K media (ATCC, Manassas, VA, USA) supplemented with $4 \mathrm{mM}$ l-glutamine, $1 \%$ penicillin, $1 \%$ streptomycin and 10\% fetal bovine serum (FBS). Cells were detached from culture with trypsin $(0.05 \%)$ and EDTA $(0.02 \%)$ and re-suspended in media for passaging to wells. As controls, human dermal fibroblasts (HDFs) were grown in DMEM supplemented with $4 \mathrm{mM}$ l-glutamine, 1\% penicillin, $1 \%$ streptomycin and 10\% FBS. Cells were detached from culture with trypsin $(0.05 \%)$ and EDTA $(0.02 \%)$ and re-suspended in media for passaging to wells.

\section{Immunofluorescence staining}

PC-3 and HDF cells were seeded on cover-slips pre-adsorbed with fibronectin $(10 \mu \mathrm{g} / \mathrm{ml})$ for $2 \mathrm{hr}$. After the cells were cultured to form a confluent monolayer, they were fixed with $4 \%$ formaldehyde for $15 \mathrm{~min}$ and then permeabilized with $0.5 \%$ triton X-100 for $5 \mathrm{~min}$. After incubating the samples with a 3\% bovine serum albumin (BSA) in PBS for $2 \mathrm{hr}$, the samples were incubated with anti-EphA2 mouse antibody (Upstate, Lake Placid, NY, USA) at a concentration of $10 \mu \mathrm{g} / \mathrm{ml}$ in $1 \%$ BSA solution for $2 \mathrm{hr}$. The samples were then incubated with anti-mouse IgG conjugated with Alexa fluor 488 (Invitrogen, Carlsbad, CA, USA) at concentration of $10 \mu \mathrm{g} / \mathrm{ml}$ in $1 \% \mathrm{BSA}$ solution for $2 \mathrm{hr}$. Cell nuclei were stained with $300 \mathrm{nM}$ DAPI (Invitrogen) for $10 \mathrm{~min}$. Controls without primary antibody were prepared in parallel. The samples were imaged with a confocal microscope (Zeiss Live 5).

\section{Cellular targeting of nanoshells}

PC-3 and HDF cells were seeded on cover-slips pre-adsorbed with fibronectin $(10 \mu \mathrm{g} / \mathrm{ml})$ for $2 \mathrm{hr}$. After 3 days in culture, the cells were incubated with nanoshells; ephrinA1-, PEG-, and bare nanoshells were suspended in media appropriate for the cell lines to a concentration of $3 \times 10^{9}$ nanoshells $/ \mathrm{ml}$. Cells were incubated with the suspensions for $2 \mathrm{hr}$ and rinsed twice with PBS. After mounting cover-slips, the samples were visualized with Axiovert 135 phase contrast microscope (Carl Zeiss, Thornwood, NJ, USA), equipped with a darkfield attachment from CytoViva (Auburn, AL, USA). Images were taken with Nikon D80 Digital SLR. For visualization of the cells and nanoshells with confocal microscopy, the cells were stained with Cell Tracker Orange CMTMR (Invitrogen) at $2.5 \mu \mathrm{M}$ for $1 \mathrm{hr}$ prior to incubation with nanoshells. The cells were visualized with confocal microscope in fluorescence mode and the nanoshells in reflectance mode.

\section{Photothermal ablation of cells}

PC-3 and HDF cells were grown in tissue culture plastic wells and incubated with either ephrinA1-, PEG-, or bare nanoshells. Nanoshells were suspended in media appropriate for the cell line at a concentration of $3 \times 10^{9}$ nanoshell $/ \mathrm{ml}$. Cells were incubated with nanoshell suspensions for $2 \mathrm{hr}$ and rinsed twice with PBS. Laser ablation was accomplished using an Integrated Fiber Array Packet, FAP-I System, with a wavelength of $808 \mathrm{~nm}$ (Coherent, Santa Clara, CA, USA) at a power density of $80 \mathrm{~W} / \mathrm{cm}^{2}$ and a spot size of $\sim 2 \mathrm{~mm}$ diameter for $7 \mathrm{~min}$.

After irradiation, cells were rinsed gently with PBS and incubated with appropriate media. After incubation for $4 \mathrm{hrs}$, cell viability was assessed using a Live/Dead stain kit from Molecular Probes (Invitrogen). Dilutions recommended by the manufacturer were followed for the staining procedure using PBS. $2.5 \mu \mathrm{l}$ of calcein AM and $8.0 \mu \mathrm{l}$ of ethidium homodimer-1 were used per $10 \mathrm{ml}$ of PBS. Cells were incubated for 45 min prior to imaging. In this stain, the calcein AM enters the cells and is cleaved by esterases in live cells to yield cytoplasmic green fluorescence. Dead cells, having compromised nuclear membranes, allow the ethidium homodimer- 1 to enter and bind nucleic acids generating a red fluorescence.

\section{Results \\ Expression of EphA2 on PC-3 cells}

EphA2 are known to be overexpressed in prostate, melanoma, breast, and lung cancers (Walker-Daniels et al 1999; Zelinski et al 2001; Hess et al 2001; Brantley et al 2002; Kinch et al 2003; Ireton and Chen 2005). In order to confirm high expression levels of EphA2 in prostate cell lines, we 
performed immunofluorescence staining of EphA2 on PC-3 cells. As shown in Figure 1A and 1C, PC-3 cells incubated with antibody against the EphA2 receptor showed strong fluorescence (green) whereas HDF had minimal fluorescence signal. There was minimal background signal as shown with PC-3 cells and HDF incubated only with the secondary antibody conjugated with fluorophores (Figure 1B and 1D).

\section{Nanoshell spectra after EphrinAI conjugation}

Nanoshells were designed to have peak absorption in the near infrared. Nanoshells were made to be $142 \mathrm{~nm}$ in diameter with a $12 \mathrm{~nm}$ thick shell and had peak absorption at $800 \mathrm{~nm}$. There was minimal spectral shift of only $\sim 5.0 \mathrm{~nm}$ of the peak at $780 \mathrm{~nm}$ after ephrinA1 or PEG conjugation to nanoshells (Figure 2). This demonstrates negligible effect of biomolecule conjugation on the optical properties of the nanoshells.

\section{Binding of EphrinA I-nanoshells to PC-3 cells}

Nanoshells are known to both absorb and scatter light at its maximal extinction wavelength (Loo et al 2005a). In order to visualize nanoshells bound to cells, confocal
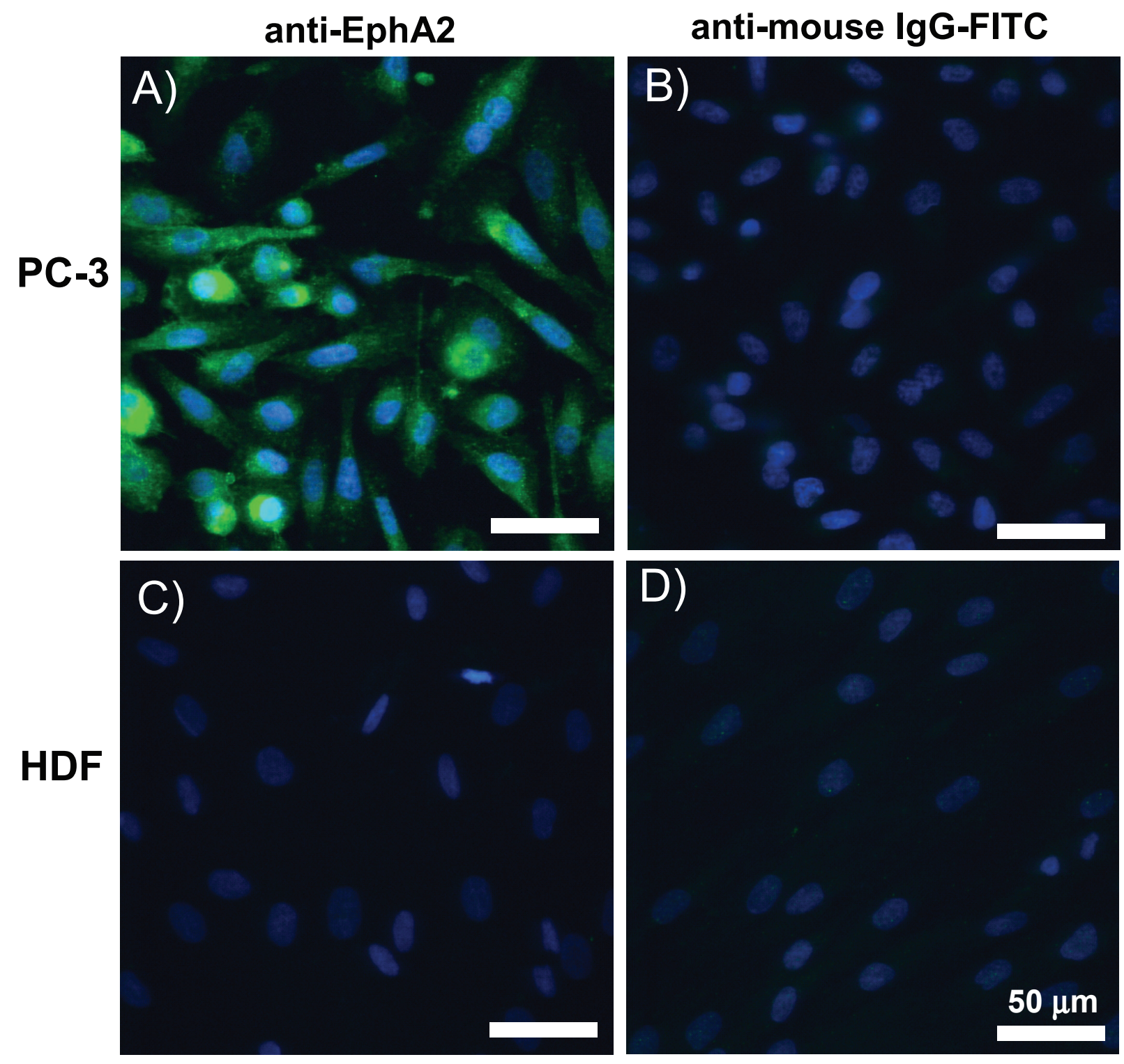

Figure I Immunofluorescence staining of human prostate cancer cells. A) A model prostate cancer cell line, PC-3, stained with anti-EphA2 antibody, shows abundant expression level of EphA2 receptor $\mathbf{C}$ ) whereas there is no detectable level in HDF cells. B), D) PC-3 and HDF cells incubated with only secondary antibody conjugated with Alex fluor 488 had minimal fluorescence background. DAPI is shown in blue. 


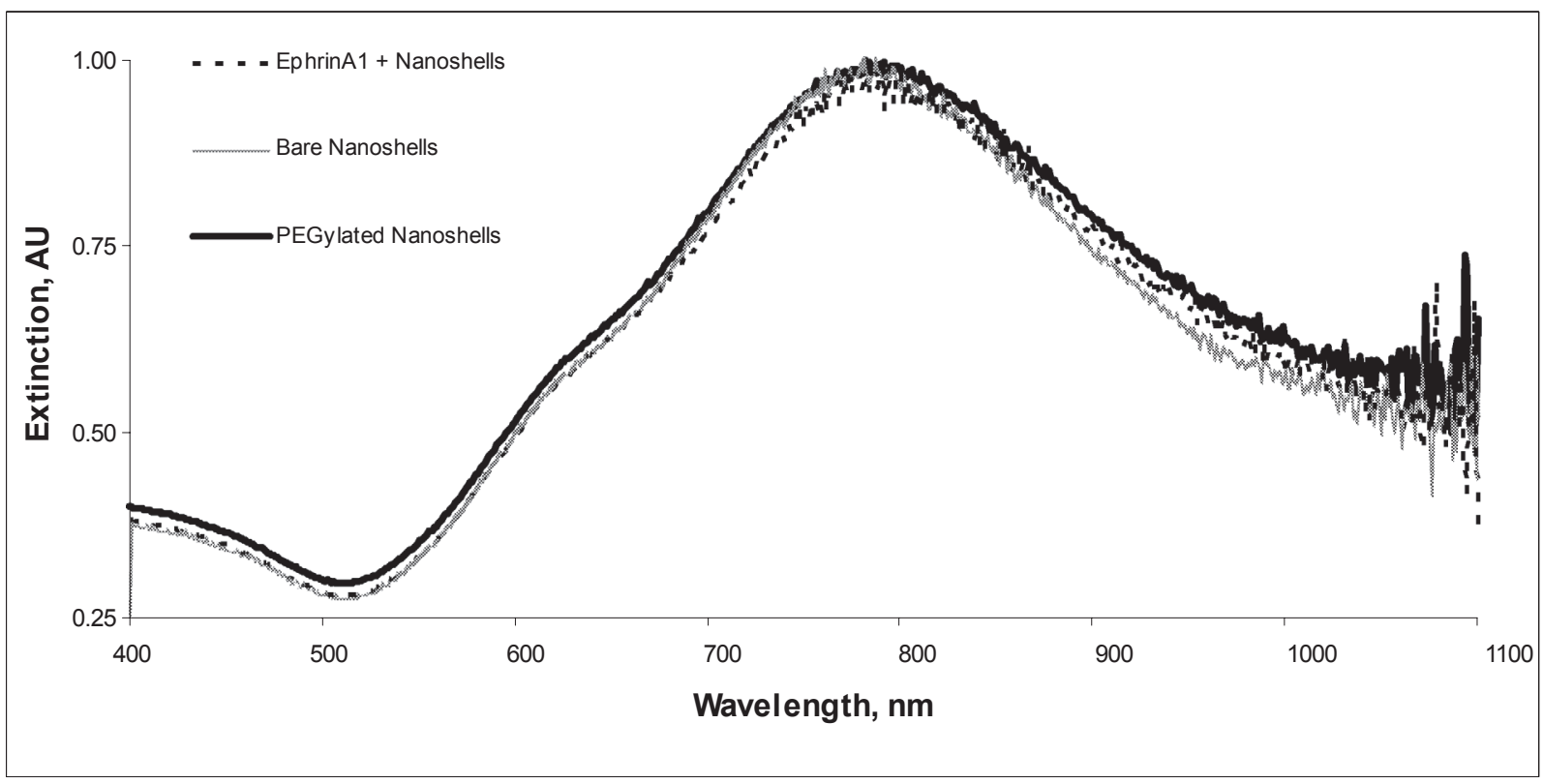

Figure 2 Spectra of nanoshells before and after surface conjugation of ephrinAI or PEG. There is minimal shift in the peak after conjugation, indicating that the nanoshells will be effective at absorbing the NIR light from the laser with minimal reduction in efficiency.

microscopy was used on PC-3 cells and HDF incubated with ephrinA1-nanoshells for $2 \mathrm{hrs}$. Images of nanoshells obtained in reflectance mode at $633 \mathrm{~nm}$ and cells pre-labeled with Cell Tracker Orange CMTMR were overlaid and shown in Figure 3. EphrinA1-nanoshells bound specifically to PC-3 cells. EphrinA1-nanoshells decorated majority of PC-3 cells whereas there was no binding of ephrinA1-nanoshells on HDF. PEG-nanoshells without the ligand for EphA2 did not bind to either cell types. On the other hand, bare nanoshells bound to both cell types as well as on fibronectin-coated glass substrates due to protein adsorption to the gold surface.

Darkfield microscopy was also used to visualize the nanoshells. Figure 4A shows ephrinA1-nanoshells bound to PC-3 cells as bright spots against the darker background of cell bodies and substrates. Images in higher magnification provide better details of the bound nanoshells (Figure 4B). There was good coverage of ephrinA1-nanoshells over the entire surface of PC-3 cells. PEG-nanoshells did not bind to PC-3 cells (Figure 4C) whereas bare nanoshells bound to both the cells and substrates nonspecifically (Figure 4D). HDF had minimal number of ephrinA1-nanoshells (Figure 4E).

\section{Targeted photothermal ablation}

EphrinA1-nanoshells bound specifically to PC-3 cells, thus allowing targeted destruction of prostate tumor cell lines with NIR light-induced photothermal ablation. As described in the methods and materials, the NIR light at $808 \mathrm{~nm}$ was applied to cells incubated with the nanoshells. Figure 5 shows cells stained with live/dead stain after treatment with the NIR laser. PC-3 cells targeted with ephrinA1-nanoshells had a zone of dead cells, corresponding to the circular pattern of the NIR laser spot (Figure 5A). However, control HDF that underwent the same treatment with ephrinA1-nanoshells and NIR laser ablation did not show any sign of cell death (Figure 5B). As expected, both cell types were viable after exposure to PEG-nanoshells and laser treatment whereas similar treatment with bare nanoshells resulted in cell death in both cell types.

\section{Discussion}

The work presented here shows that PC-3 cell line can be specifically targeted and destroyed by nanoshells conjugated to ephrinA1. We first confirmed previous reports of increased expression level of EphA2 receptor in prostate cancer cell lines by immunofluorescence staining. High levels of the EphA2 receptor in PC-3 cells allowed selective binding and accumulation of ephrinA1 conjugated nanoshells over a control cell line, HDF, which had minimal level of EphA2 expression. Using both reflectance confocal microscopy and darkfield microscopy, we have visualized ephrinA1nanoshells decorating surface of PC-3 cells. Finally, PC-3 cells incubated with ephrinA1-nanoshells were destroyed with NIR laser matched to the peak resonance of the nanoshells. 

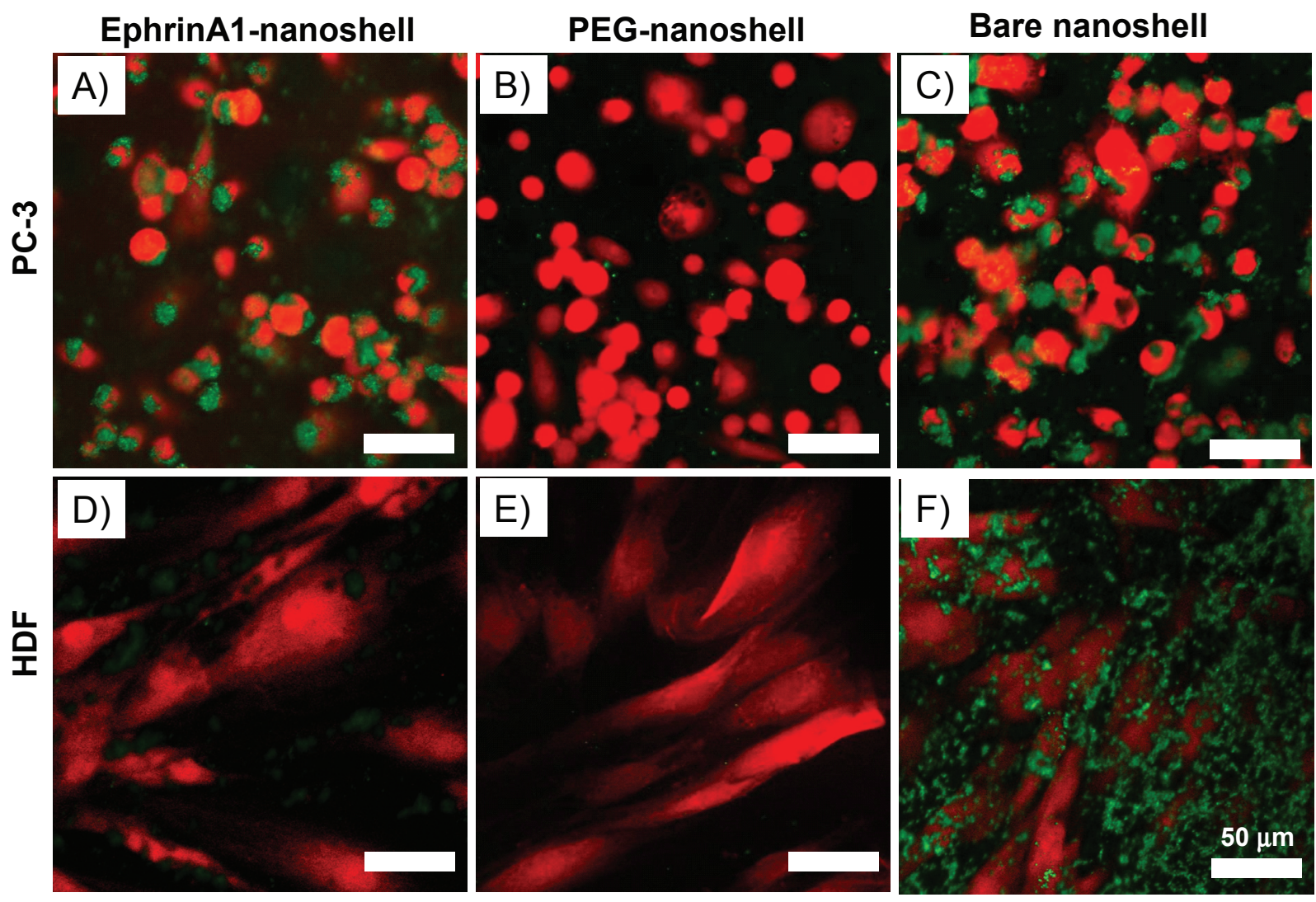

Figure 3 Reflective confocal images of nanoshells bound to cells. PC-3 cells pre-labeled with Cell Tracker Orange were incubated with A) ephrinAI-, B) PEG-, and C) bare nanoshells and visualized with confocal microscopy. Surface-bound nanoshells are shown in green.A large number of ephrinAI-nanoshells and bare nanoshells bound to PC-3 cells. On the other hand, D), E), F) only bare nanoshells associated with the control HDF cells.

Nanoshell therapy possesses several advantages over chemotherapy or radiation therapy. First, the use of the goldcoated nanoshells does not present a danger to the patient in terms of toxicity and overall side effects. Secondly, the nanoparticles can passively accumulate to the tumor based on the intrinsic biology of the tumor. Third, the use of targeted nanoshells should increase localized accumulation and allow specific targeting of malignant cells over the normal tissues. Finally, the nanoshells will only heat up and destroy tumors in the presence of NIR light activation, thus localizing treatment to the exact site needed.

The therapeutic benefits of nanoshells are realized after the particles are heated by absorption of NIR laser energy. Neither the nanoshells nor the NIR laser by themselves produce any damaging effects to the tissue; it is the combination of the two within the tumors that produce hyperthermia and thus ablation of the tumor. Solid gold nanoparticles do not induce cytotoxicity (Connor et al 2005), whereas chemotherapeutic agents produce systemic side effects while treating certain tumors.

Energy absorption by tissues in NIR window is minimal (Weissleder 2001). Thus, NIR laser irradiation permits deep penetration of the laser energy into tumor, and heat generated from nanoshells irradiated with NIR raises the local temperature, causing irreversible thermal damage. Two innocuous components, nanoshells and NIR laser irradiation, can be combined to produce an effective therapy, minimizing harmful effects to the patient. The ability of the nanoshell to both absorb and scatter energy based on its design has permitted imaging of tumors using optical methods (Gobin et al 2007), and targeted nanoshells hold the potential for simultaneous imaging and treatment of cancers (Loo et al $2005 b)$. In the treatment of aggressive prostate cancer, these types of options will provide an improved therapeutic since targeted nanoshells will allow surgeons to locate the malignant cells and destroy them locally and specifically.

This study used a prostate cancer cell line as a tumor model to demonstrate the efficacy of using ephrinA1 as a targeting moiety in nanoshell therapy. Promising results from this study suggests that EphA2 receptor may serve as a targeting moiety in other types of tumor such as melanoma, breast and lung cancers with high expression levels of EphA2 (Walker-Daniels et al 1999; Zelinski et al 2001; 

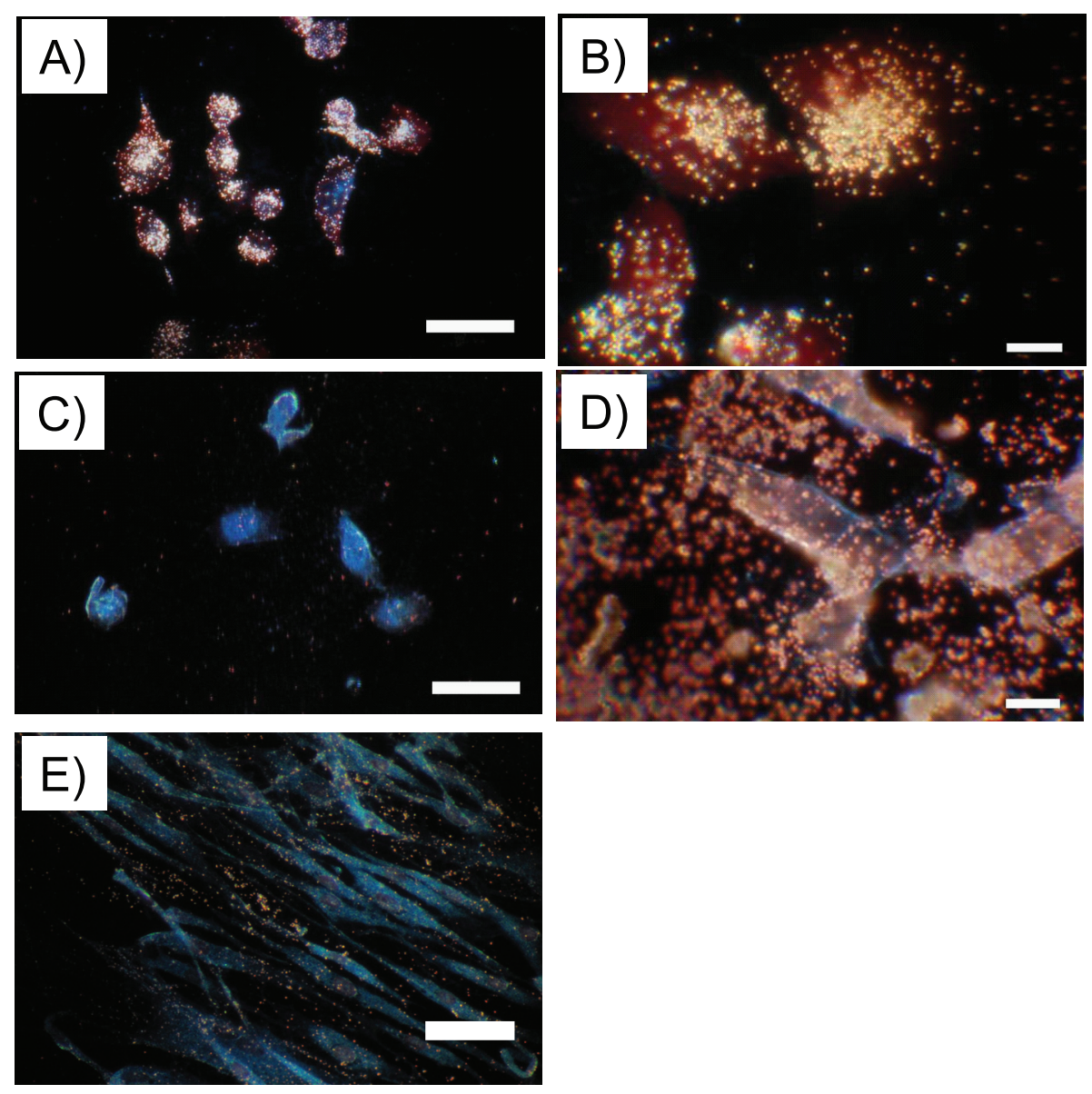

Figure 4 Darkfield images of nanoshells bound to cells. A) EphrinAI-nanoshells bound to the PC-3 cells with high expression of EphA2 receptor. B) shows higher magnification of A). C) PEG-nanoshells had minimal binding whereas D) bare nanoshells bound indiscriminately to PC-3 cells and the substrate. E) EphrinAI-nanoshells had minimal binding to HDF. Scale bars in $\mathbf{A}), \mathbf{C})$ and $\mathbf{E})=50 \mu \mathrm{m}$ and $\mathbf{B}$ ) and $\mathbf{D})=10 \mu \mathrm{m}$.
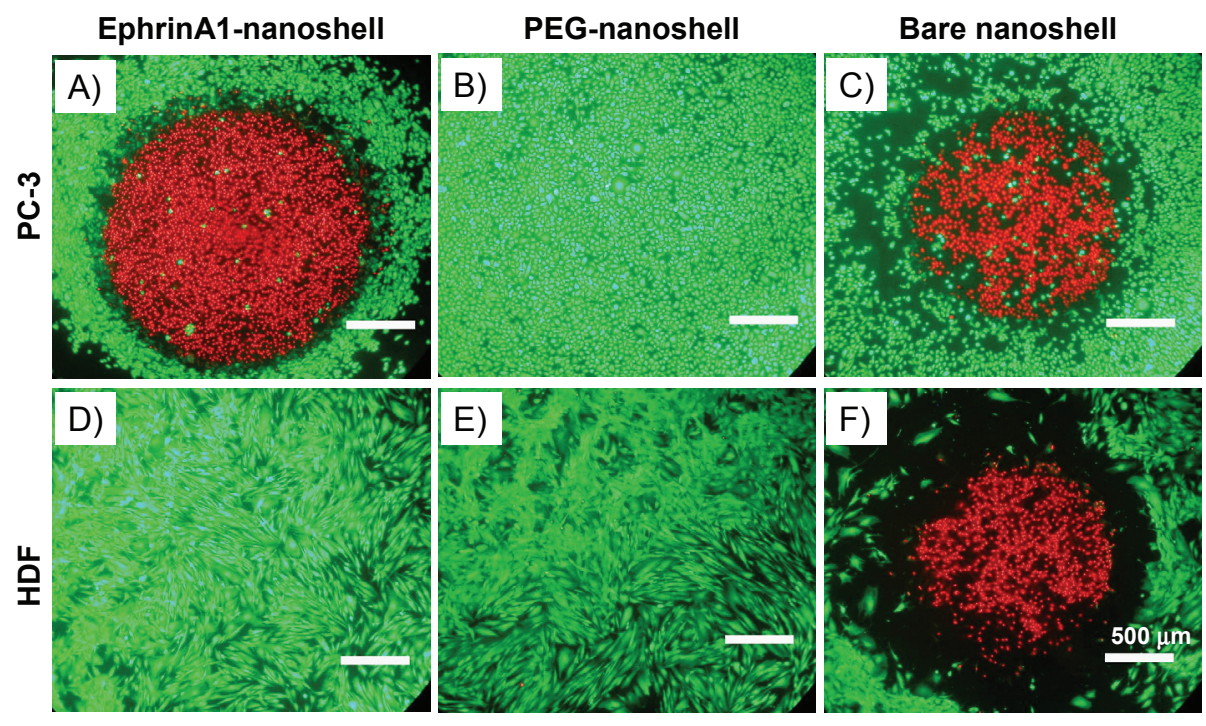

Figure 5 Live/dead stain of cells incubated with nanoshells and exposed to the NIR laser with a spot size of $2 \mathrm{~mm}$. PC-3 cells were incubated with A) ephrinAI-, B) PEG-, and $\mathbf{C}$ ) bare nanoshells for 2 hrs and then exposed to the NIR laser in the center of the wells, and $\mathbf{D}$ ), E), and F) HDF underwent the same treatment. Note that the bare nanoshells allowed ablation of both cell types indiscriminately while ephrinAI-nanoshells allowed ablation of only PC-3 cell line. Live and dead cells are shown in green and red, respectively. 
Hess et al 2001; Brantley et al 2002; Kinch et al 2003; Ireton and Chen 2005). In addition, a large percentage of cells within these tumors are known to overexpress EphA2 receptor, and the most aggressive tumor cells show the highest levels of EphA2 (Fox and Kandpal 2004; Fox et al 2006). Therefore, nanoshells targeting EphA2 via ephrinA1 ligand may be an excellent candidate for targeting and destroying a wide variety of tumors.

This work demonstrated that we can achieve good conjugation of biomolecules specifically targeted for prostate cancer cells on nanoshells without significant changes in their spectral properties. EphA2 receptor protein overexpressed on surface of prostate cancer cells were targeted by its complimentary ligand, ephrinA1, conjugated onto nanoshells. PC-3 cells bound with these nanoshells were destroyed when laser energy benign to normal tissue was applied. It is expected that this targeting scheme should enhance the uptake of nanoshells to tumors and thus increase the efficacy of the nanoshell therapy in vivo.

\section{Disclosure}

Andre M Gobin and James J Moon contributed equally to this work. This work was funded by NIH and NSF. The authors report no conflicts of interest.

\section{References}

Averitt RD, Westcott SL, Halas NJ. 1999. Linear optical properties of gold nanoshells. J Optic Soc Am B, 16:1824-32.

Brantley-Sieders D, Schmidt S, Parker M, et al. 2004. Eph receptor tyrosine kinases in tumor and tumor microenvironment. Curr Pharm Des, 10:3431-42.

Brantley DM, Cheng N, Thompson EJ, et al. 2002. Soluble Eph A receptors inhibit tumor angiogenesis and progression in vivo. Oncogene, 21:7011-26.

Carles-Kinch K, Kilpatrick KE, Stewart JC, et al. 2002. Antibody targeting of the EphA2 tyrosine kinase inhibits malignant cell behavior. Cancer Res, 62:2840-7.

Connor EE, Mwamuka J, Gole A, et al. 2005. Gold nanoparticles are taken up by human cells but do not cause acute cytotoxicity. Small, 1:325-7.

Duff DG, Baiker A, Edwards PP. 1993. A new hydrosol of gold clusters. 1. Formation and particle size variation. Langmuir, 9:2301-9.

Fang WB, Brantley-Sieders DM, Parker MA, et al. 2005. A kinasedependent role for EphA2 receptor in promoting tumor growth and metastasis. Oncogene, 24:7859-68.
Fox BP, Kandpal RP. 2004. Invasiveness of breast carcinoma cells and transcript profile: Eph receptors and ephrin ligands as molecular markers of potential diagnostic and prognostic application. Biochem Biophys Res Commun, 318:882-92.

Fox BP, Tabone CJ, Kandpal RP. 2006. Potential clinical relevance of Eph receptors and ephrin ligands expressed in prostate carcinoma cell lines. Biochem Biophys Res Commun, 342:1263-72.

Gobin AM, Lee MH, Halas NJ, et al. 2007. Near-infrared resonant nanoshells for combined optical imaging and photothermal cancer therapy. Nano Lett, 7:1929-34.

Hess AR, Seftor EA, Gardner LM, et al. 2001. Molecular regulation of tumor cell vasculogenic mimicry by tyrosine phosphorylation: role of epithelial cell kinase (Eck/EphA2). Cancer Res, 61:3250-5.

Hirsch LR, Jackson JB, Lee A, et al. 2003. A whole blood immunoassay using gold nanoshells. Anal Chem, 75:2377-81.

Ireton RC, Chen J. 2005. EphA2 receptor tyrosine kinase as a promising target for cancer therapeutics. Curr Cancer Drug Targets, 5:149-57.

Kinch MS, Moore MB, Harpole DH, Jr. 2003. Predictive value of the EphA2 receptor tyrosine kinase in lung cancer recurrence and survival. Clin Cancer Res, 9:613-18.

Loo C, Hirsch L, Lee M-H, et al. 2005a. Gold nanoshell bioconjugates for molecular imaging in living cells. Opt Lett, 30:1012-14.

Loo C, Lowery A, Halas N, et al. 2005b. Immunotargeted nanoshells for integrated cancer imaging and therapy. Nano Lett, 5:709-11.

Lowery AR, Gobin AM, Day ES, et al. 2006. Immunonanoshells for targeted photothermal ablation of tumor cells. Int J Nanomed, 1:149-54.

Marston DJ, Dickinson S, Nobes CD. 2003. Rac-dependent transendocytosis of ephrinBs regulates Eph-ephrin contact repulsion. Nat Cell Biol, 5:879-88.

Miao H, Burnett E, Kinch M, et al. 2000. Activation of EphA2 kinase suppresses integrin function and causes focal-adhesion-kinase dephosphorylation. Nat Cell Biol, 2:62-9.

O'Neal DP, Hirsch LR, Halas NJ, et al. 2004. Photo-thermal tumor ablation in mice using near infrared-absorbing nanoparticles. Cancer Lett, 209:171-6.

Oldenburg SJ, Averitt RD, Westcott SL, et al. 1998. Nanoengineering of optical resonances. Ch Phys Lett, 288:243-7.

Poliakov A, Cotrina M, Wilkinson DG. 2004. Diverse roles of eph receptors and ephrins in the regulation of cell migration and tissue assembly. Dev Cell, 7:465-80.

Surawska H, Ma PC, Salgia R. 2004. The role of ephrins and Eph receptors in cancer. Cytokine Growth Factor Rev, 15:419-33.

Walker-Daniels J, Coffman K, Azimi M, et al. 1999. Overexpression of the EphA2 tyrosine kinase in prostate cancer. Prostate, 41:275-80.

Weissleder R. 2001. A clearer vision for in vivo imaging. Nat Biotechnol, 19:316-7.

Zelinski DP, Zantek ND, Stewart JC, et al. 2001. EphA2 overexpression causes tumorigenesis of mammary epithelial cells. Cancer Res, 61:2301-6.

Zeng G, Hu Z, Kinch MS, et al. 2003. High-level expression of EphA2 receptor tyrosine kinase in prostatic intraepithelial neoplasia. Am J Pathol, 163:2271-6. 